Article

\title{
Generation and Mitigation of Conducted Electromagnetic Interference in Power Converters - a Big Picture
}

\author{
Arnold de Beer \\ Department of Electrical and Electronic Engineering Science, University of Johannesburg, Johannesburg, \\ South Africa; asdebeer@uj.ac.za
}

\begin{abstract}
This article is a big picture of how electrical noise or conducted Electromagnetic Interference (EMI) is generated and mitigated in power converters. It gives an overview of what EMI in power converters is - from generation through to conduction and mitigation. It is meant to cover the complete subject as a summary so that the reader will have an outline of how to control conducted EMI by design (where possible) and how to mitigate by filtering. A clear distinction is made between Differential Mode (DM) and Common Mode (CM) EMI generation and mitigation. By using a boost converter as an example the trade-offs for DM noise control are discussed. It is shown how CM EMI is generated in a boost converter using the concept of the "Imbalance Difference Model" (IDM). Practical measurements for an in-line power filter is given showing the effect of the filter on the total EMI of a boost converter. Measurements for the CM current produced due to the imbalance difference for different values of the boost conductor are also shown. The main contribution of this study is linking CM noise generation to DM EMI. It is shown that CM noise is a direct consequence of DM noise (although circuit imbalance and coupling to a common ground also play a role). This paper will be useful to designers seeking the "bigger picture" of how EMI is generated in power converters and what can be done to mitigate the noise.
\end{abstract}

Keywords: Power Converters, Power Electronics, Electromagnetic Interference; EMI, Noise, Differential Mode, DM, Common Mode, CM, Imbalance Difference Model, Boost Converter

\section{Introduction}

Power converters (consisting of power electronics) play an important role in modern day electrical and electronic systems. They are electric energy conversion devices. They convert Alternating Current (AC) or Direct Current (DC) to different voltage levels (and frequency) of AC and/or DC. Examples are:

- Power supplies where $50 \mathrm{~Hz}$ or $60 \mathrm{~Hz}$ from the utility grid is converted to DC. Most consumer electronics have a circuit for this.

- Inverters, where DC (typically from battery level voltage) is converted to $50 \mathrm{~Hz} / 60 \mathrm{~Hz} \mathrm{AC}$.

- DC-to-DC converters which are used inside systems to transform one DC voltage level to another.

Power converters are an enabling technology [1]. This means that they are not necessarily part of the functional section of a system, but rather are adjunct to the main function. They support and supply power without which no system can operate. As such they are found in all electronic systems and products. As technology progresses towards the future it has been predicted that power conversion will play pivotal roles in the Smart Grid [2],[3] and Internet of Things [4], [5],[6],[7].

Power converters used to be linear [8] (p. 4). This resulted in large losses and physically large systems - mostly due to the area necessary to get rid of the excess heat. Although still used in special applications, linear power conversion has mostly been replaced by switching converters.

Switching converters are a fraction of the size of linear converters and markedly more efficient. There is however a caveat. Because of the switching action, all switch mode power electronics 
produce electrical noise. This is also known as Electromagnetic Interference (EMI). The reason it is called interference is because the electrical noise can either interfere with the operation of the device generating the noise, or it can interfere with other systems in close proximity to the noise producing culprit.

The production of EMI is due to the fact that modern power converters use switching electronics (transistors) to convert voltages and power levels. These converters employ either transformers and/or capacitors as well as inductors to boost, reduce or isolate electric power at the switching frequency. The switching frequency is much higher than the DC or AC being converted. There are numerous topologies of converters - all employing a switching frequency. The reader is referred to one of numerous textbooks on different converters and topologies [9].

At this stage it is important to understand the relationship between the main power transfer through a power electronics converter and the direction of the noise energy flow. Figure 1 gives a block diagrammatic representation of a power electronics converter and the direction of converted electric energy flow vs. that of the noise being created. The power source can typically be the $50 \mathrm{~Hz}$ grid and the load an electric machine. In this example the converter act as a variable speed drive and outputs typically a variable frequency AC up to $100 \mathrm{~Hz}$. The main electric energy flow is therefore from left to right. The noise (EMI) however, is generated inside the converter (at $\mathrm{kHz}$ switching frequencies) and permeates towards the power source and the load. At the power source or the load the EMI can interfere with sensitive electronics or the noise can affect the operation of the power electronics converter itself.

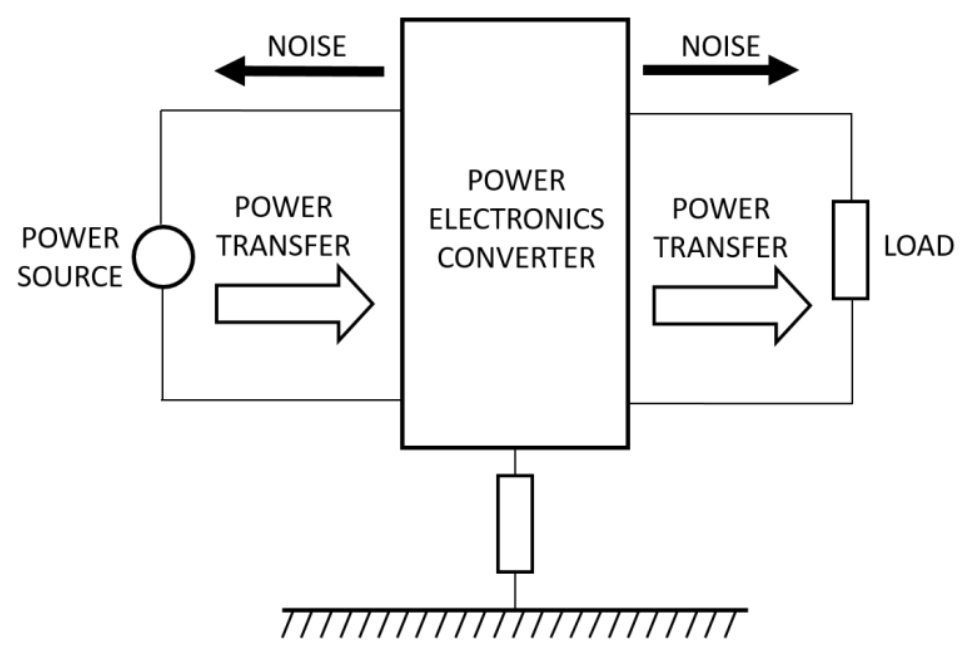

Figure 1. Block diagrammatic representation of a power electronics converter and the direction of converted electric energy flow $v s$. that of the noise being created.

It is not uncommon for power electronic converters to share a common power source. This common source must be protected from converter EMI (noise). To this effect different regulations and standards are mandatory for converters and regulate the noise contribution of a converter. Power line filters (see Figure 5) are used to block and divert noise from the converter so that EMI cannot reach the power source. In Figure 1 a power line filter is usually implemented on the left hand side of the converter. This block or shunt EMI from reaching the common supply. It is left to the manufacturer to control the amount of load EMI (on the right hand side of Figure 1) to a level acceptable for internal product operation.

Figure 1 shows the converter coupled trough an impedance to a chassis. This is to emphasize that EMI and noise generation not only takes place in the "normal" Differential Mode (DM) circuit but also has a relationship to a common ground in Common Mode (CM). These two modes are dealt with in this paper.

In section 2 of this work, definitions for DM and CM are presented. It is also shown how total EMI (the sum of DM and CM EMI in the time domain) is used for compliance testing. In section 3, DM EMI generation and mitigation (reduction) are dealt with by using the example of a common 
fundamental converter topology - the boost converter. The boost converter is only used as an example as the fundamentals presented in this study are applicable to any power converter. Section 4 deals with the generation and mitigation of CM EMI. Once again using the boost converter as an example.

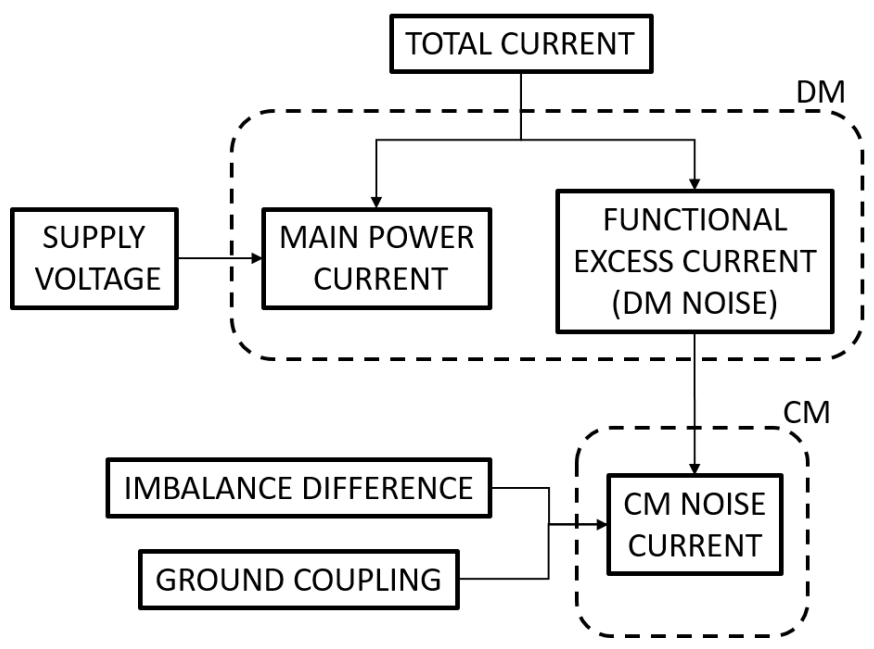

Figure 2. Block diagrammatic representation of the relationship between total, DM and CM current with influencing factors.

Figure 2 is an overview of the relationships between total current, DM and CM current with influencing factors. It summarises the generation of EMI as presented in this paper.

Assume that a power source is supplying a power converter. There will be a total current flow from source to converter (such as in the left hand side of Figure 1). This is the "Total Current" at the top of Figure 2. Most of the DM current is used to transport energy from source to converter and is influenced by the supply voltage wave shape [10]. The EMI usually presents itself as a ripple at the switching frequency superimposed on the main supply current. Both the "main power current" and "functional excess current" are in DM. The reason the DM noise is called "functional excess current" is that this is the ripple the converter produce in order to operate (see sections 2.1. and 3.1.).

Figure 2 also shows the CM current and the factors influencing conversion from DM EMI to CM EMI (see section 4). DM noise directly drives CM noise but is dependent on the "imbalance difference" of the CM circuit. It also depends on the coupling of the CM circuit to a common "ground". This is covered in section 4 .

\section{Differential and Common Modes}

This section explains and gives definitions for DM, CM and total EMI. In the first two subsections below and in Figure 3 and Figure 4 noise is shown emanating from the converter "flowing" through to the main power source. This represents a typical practical problem where the converter "pollutes" the supply grid. As per Figure 1, one should not confuse the main energy flow of the conversion process and the EMI. The EMI or noise is superimposed on the power carrying signal and can be thought of as originating in the converter and "flowing" towards the source.

\subsection{Differential Mode Noise}

Total DM current flows between a source and a power electronics converter as shown in Figure 3. In this mode current flows on the positive and negative lines (live and return) isolated from a common ground (like a power supply chassis). DM current flow is per the normal functional circuit. The DM current $I_{D M}(t)$ consists out of two sub components or:

$$
I_{D M}(t)=\frac{v_{\text {SOURCE }}(t)}{R_{\text {CONVERTER }}}+i_{D M_{-} E M I}(t) .
$$


- The first sub-component in (1) is the energy carrying current flow to the converter that returns to the source or $\frac{v_{\text {SOURCE }}(t)}{R_{\text {CONVERTER }}}$. This is the "main power current" in Figure 2 . The main power current to a converter is influenced by the source or main functional voltage $\left(v_{\text {SOURCE }}(t)\right.$ in $\left.(1)\right)$. This is due to the fact that nett electrical energy can only be transferred by a scaled version of the source voltage [10]. Another way of stating this is that for electric energy transfer the source side input impedance to a power electronics converter must be the equivalent of a resistor $\left(R_{\text {CONVERTER }}\right.$ in (1)). This fact is again used in Section 3 when pairing a converter with a filter.

- The second sub-component in (1) is DM EMI $\left(i_{D M_{-} E M I}(t)\right)$ and is electrical noise that is superimposed on the power carrying signal. This is the high frequency switching ripple on the usually $50 \mathrm{~Hz} / 60 \mathrm{~Hz}$ or DC supply. Both the "main power current" and "functional excess current" of Figure 2 are in DM. The reason the DM noise is called "functional excess current" is that this is the ripple the converter produce in order to operate (see section 3.1.).

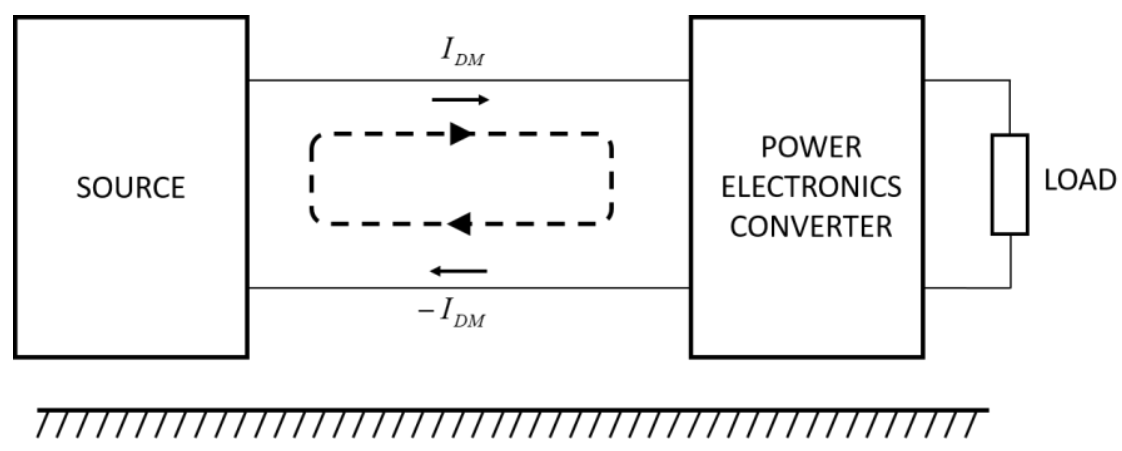

Figure 3. Representation of differential mode current flow between a power electronics converter and the source.

\subsection{Common Mode Noise}

DM noise current flows in circuits in functional current paths. However, CM current flow is parasitic. CM current flow between a source and a power electronics converter is shown in Figure 4 . The current path for the CM flow shares a common ground (or typically a chassis). The current path consist of parasitic components (usually capacitive) that complete the path between the active circuitry and a common ground.

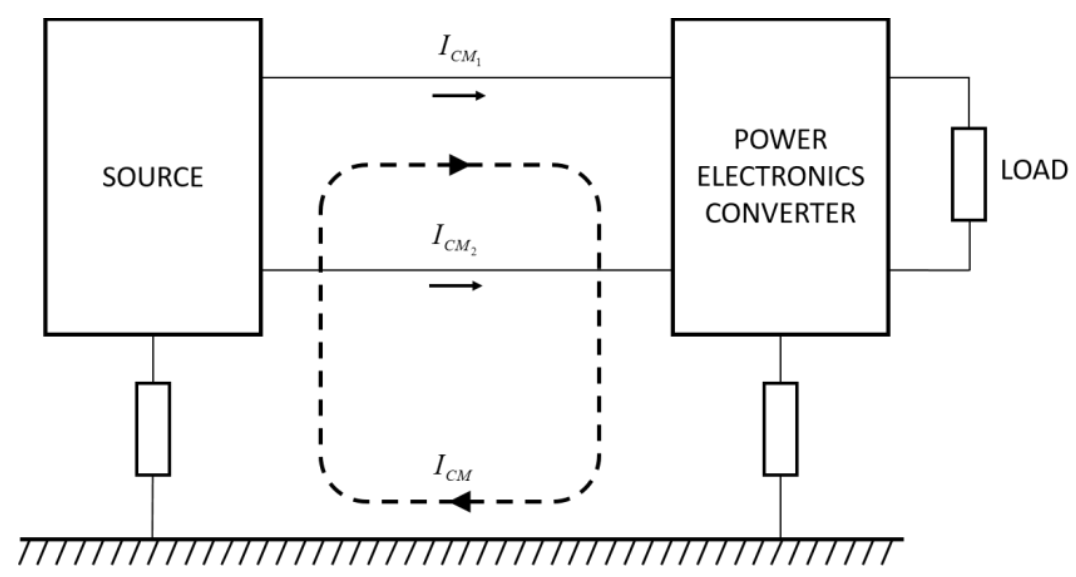

Figure 4. Representation of common mode current flow between a power electronics converter and the source.

As can be seen in Figure 4, the CM current flowing on the positive and negative (live and return) is in the same direction but does not necessarily split equally between the two connections. In general: 


$$
I_{C M}=I_{C M_{1}}+I_{C M_{2}} \text {. }
$$

\subsection{Measurement and Total EMI}

All electronic power converters must conform to international and local EMI standards and regulations. Their EMI signature must be below a given minimum so that they do not interfere with other electronic systems in their electric and electromagnetic environment. In order to ensure this, a standardised measurement setup is used to measure total conducted EMI (usually in the range 150 $\mathrm{kHz}-30 \mathrm{MHz}$ ).

Note that for conformance, no distinction is made between DM and CM EMI. The standardised measurement setup includes a device called a Line Impedance Stabilisation Network or LISN. Figure 5 shows a power electronics converter connected to a source (either DC or $50 \mathrm{~Hz} / 60 \mathrm{~Hz}$ ) and a high frequency (HF) model of a LISN and the position of a power line filter. The power line filter shorts and then block noise from the converter and is explored further in sections 3 and 4 . It is shown here for reference.

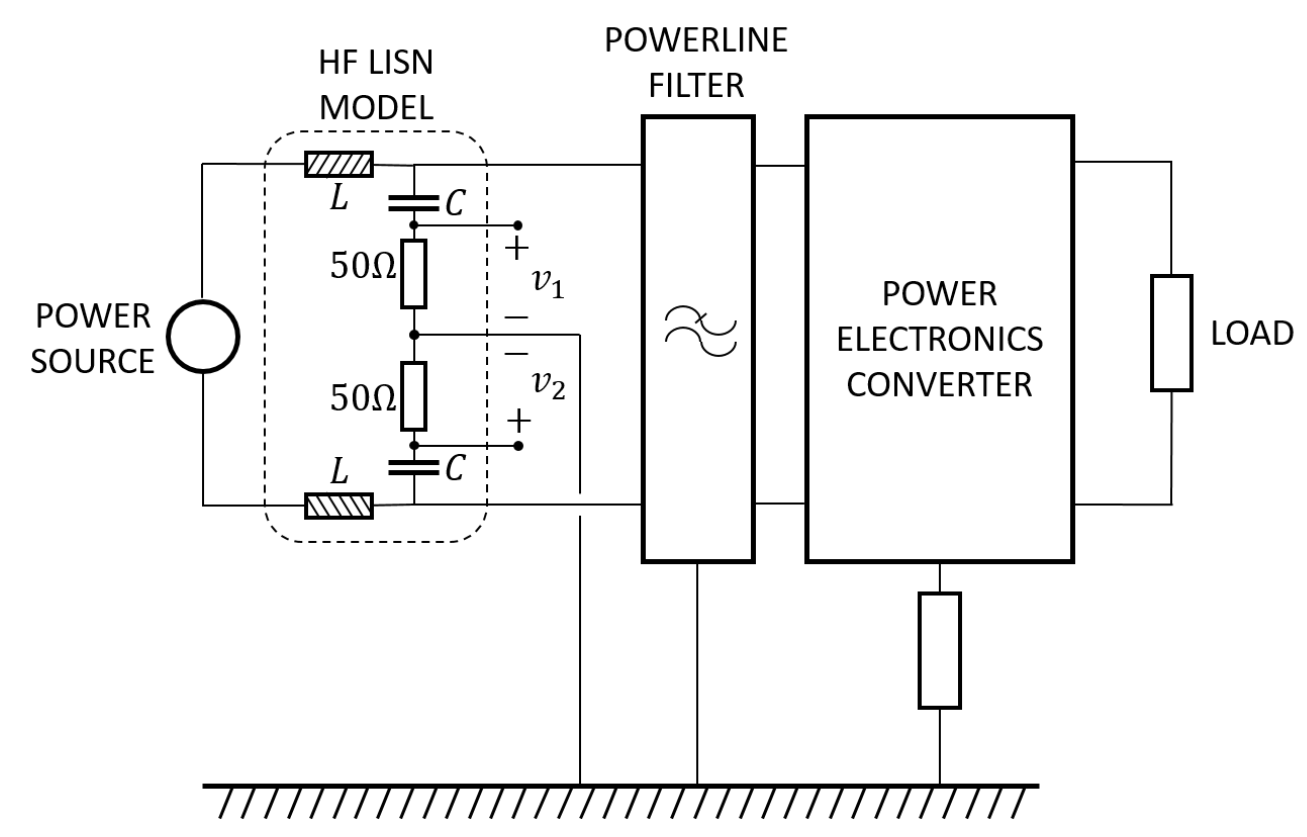

Figure 5. Power electronics converter connected to a source, LISN and filter for the measurement of total EMI.

Operation of the LISN can be described as follows:

- At low frequencies the impedance of $L$ is low and $50 \mathrm{~Hz} / 60 \mathrm{~Hz} \mathrm{AC}$ or DC can pass from the Power Source (in Figure 5) to supply the converter.

- From the converter side, the LISN presents a standardised load impedance for the noise generated by the power electronics. This noise is at higher frequencies than the supply from the power source, due to the switching frequency. At sufficiently high frequencies, $L$ will block the generated EMI, $C$ will have a low impedance and the noise load is presented as $50 \Omega$.

- Measurements are made across $50 \Omega$ for the total EMI as both DM and CM noise from the converter flows through the $50 \Omega$. The voltage $v_{l}$ represents the total EMI on the positive or live line while $v_{2}$ represents the total EMI on the negative or return line.

At higher frequencies the noise current flows through the $50 \Omega$ and two voltages are measured that represent the total EMI or:

$$
v_{1,2}=50 \cdot\left(I_{D M_{1,2}}+I_{C M_{1,2}}\right)
$$


These two voltages $v_{1}$ and $v_{2}$ are then subjected to limits from an appropriate regulation or standard.

It should be noted that $v_{1}$ and $v_{2}$ are representations of the total EMI and only valid at high frequencies (typically higher than $1 \mathrm{MHz}$ ). There are some controversy surrounding the true EMI of measured converters (using a LISN) at low frequencies [11] but for the purpose of this study it is assumed that by reducing DM and CM EMI, the values for $v_{l}$ and $v_{2}$ are reduced and therefore the total EMI at all frequencies.

\section{Differential Mode in a Boost Converter}

\subsection{Generation of Differential Mode EMI}

Differential Mode EMI in a boost converter is studied in the absence of any coupling to a common ground (or chassis). To understand DM generation in a boost converter the operation of the converter must first be introduced. This is done with the aid of Figure 6 - which is the simplest form of a boost converter and is assumed to be in continuous current mode [8].

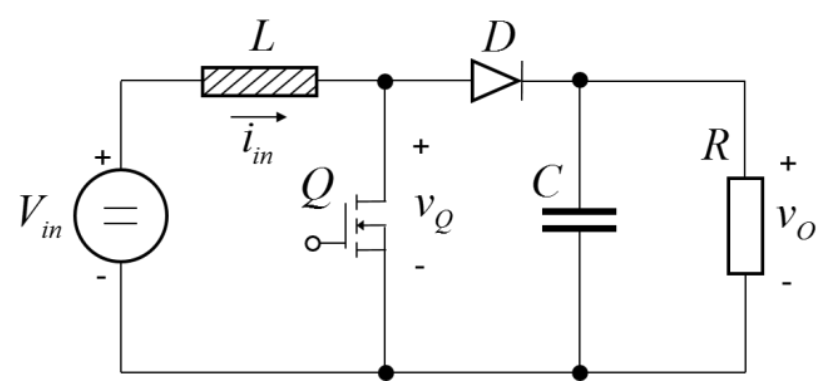

Figure 6. Functional circuit of a Boost Converter (DM).

The converter boosts a DC input voltage ( $V_{\text {in }}$ in Figure 6) to a higher output or load voltage $v_{o}$. The output is also DC but with ripple on it (see Figure 7). $R$ in Figure 6 represents a load. Ripple on the output $v_{o}$ is reduced by the size of $C$. The boosting action is provided by switching a power electronic switch (such as a MOSFET " $Q$ ") to charge $L$. By turning the switch $(Q)$ off, the voltage across $L$ climbs and is transferred to the load side by the diode $D$. The output voltage is determined by the input voltage and the duty cycle of the switching action, or:

$$
v_{o}=\frac{1}{1-D} V_{\text {in }} \text {, }
$$

where $1 /(1-D)$ is the DC gain of the boost converter and $D$ the duty cycle.

The switching typically has a frequency in the tens of $\mathrm{kHz}$ to low $\mathrm{MHz}$. It is this switching action that leads to DM EMI. 


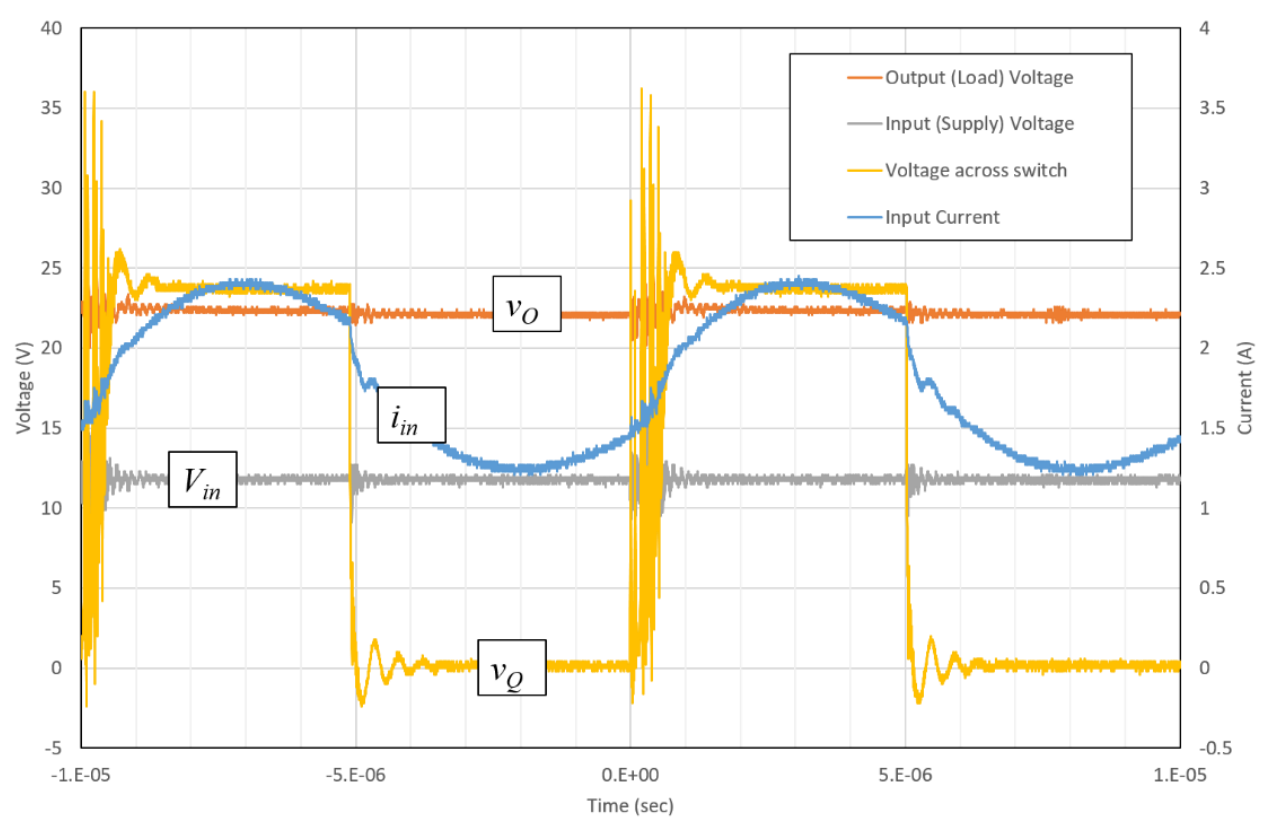

Figure 7. Measured boost converter time domain waveforms (in DM) for the conceptual circuit of Figure 6 - showing the switching action in continuous current mode.

As can be seen in the practical measurements of Figure 7, the input current $\left(i_{i n}\right)$ is not only DC. It contains a DC component with a shaped wave superimposed on the average (or DC). A representation of the harmonics for this input current is shown in Figure 8 (as seen by the LISN). The frequency domain representation of $i_{i n}$ contains a DC component and harmonics spaced at the switching frequency $f_{s}[12]$. The exact shape and outline of the harmonics vary with operating conditions. The harmonics however, diminish at higher frequencies but it should be noted that the input current contains numerous high frequency components. It is these numerous high frequency input current components that are seen as noise or EMI.

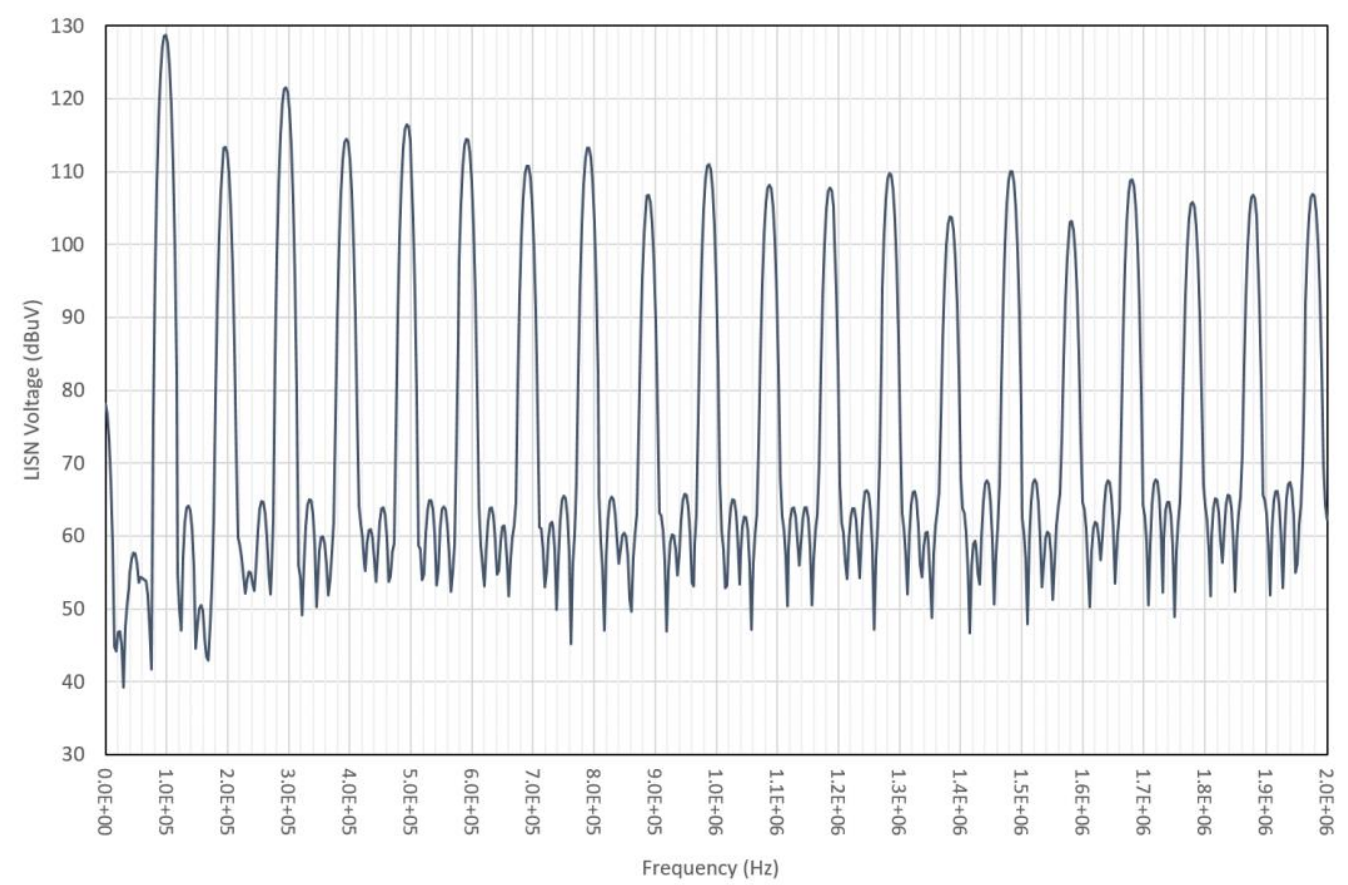

Figure 8. Measured frequency harmonics of the input current $i_{\text {in }}$ of a simple boost converter (Figure 6) at multiples of the switching frequency. In this case the switching frequency is close to $100 \mathrm{kHz}$. 
The term "DM noise" or "DM EMI" for the input current of a boost converter is however ambiguous. Although the high frequency DM components seem to be noise it can be argued that this current wave shape is functional. The majority of the power being converted is drawn from to input or supply voltage's DC component [10] but the higher frequency components are due to and necessary for the switching action. In Figure 2, the DC component is described as the "Main Power Current" and the rest of the components as "Functional Excess Current". It is this "Functional Excess Current" that is also known as EMI or power line noise.

In order to reduce total EMI, DM and CM noise currents need to be reduced. Especially DM, as $\mathrm{DM}$ is not only noise on its own, but interference that converts to or drive the CM mechanisms. Total EMI reduction therefore starts with reduction in DM.

In this section it is shown that:

- Within limits, the switching frequency (and therefore DM EMI signature) can be shifted

- The Boost Inductor can be made large as to reduce DM EMI, but there are steady state response limits to this and

- An LC-filter reduces DM EMI but it size is limited due to the possibility of converter instability and possible lack in high frequency load response.

\subsubsection{Altering DM EMI by shifting the switching frequency}

Technically not reducing DM EMI, but a shift in the interference spectrum can be introduced by choosing a suitable switching frequency $f_{s}$. In a practical converter however, switching frequency is chosen and limited to not being too low as to generate audible noise and not too high as to overheat - as switching losses in a converter are directly proportional to $f_{s}$ [8]. Within these practical constraints, the frequency of the harmonics in Figure 8 can be influenced by choosing $f_{s}$. Frequency spectrum shifting is used to avoid generating EMI that interfere with other systems that is sensitive to a very specific frequency.

\subsubsection{Reducing DM EMI by increasing the boost inductor}

Power electronic converters use switching elements (usually transistors) together with electromagnetic storage elements (inductors and capacitors) to shift electrical energy in voltage level and frequency. Different switching topologies are used. This switching usually results in current and voltage ripple which is seen as noise. In the case of the boost converter, the input boost inductor determines the input current ripple and the magnitude of the resultant EMI.

The ripple content of $i_{i n}$ in Figure 7 can be reduced if the boost inductor size $L$ is increased. This in turn will reduce the harmonic content shown in Figure 8 and therefore the DM EMI. This will further decrease the CM EMI and total EMI. Following this thought of reason, one can argue that $L$ must be as large as possible. In fact, if $L$ is infinitely large, $i_{\text {in }}$ will be perfect DC with no ripple and no EMI. This however is not practical as $L$ (and $C$ ) determine the transitory response of the converter with regards to three cases [13]:

- The response of the output $v_{o}$ to variation in input $V_{\text {in }}$;

- $\quad$ The response of the output $v_{o}$ to variation in load $R$;

- The response of the output $v_{o}$ to variation in the set point of the voltage gain or the duty cycle $D$.

\subsubsection{Reducing DM EMI by Filtering}

This sub-section introduces reduction of DM EMI by filtering. From a practical point of view a power electronics converter rarely conforms to EMI limits and standards without filtering. Complete papers and textbooks are dedicated to the design of power line filters [14], [15], [16]

First an idealised shunt current compensator is dealt with and secondly a conventional LC-filter. 


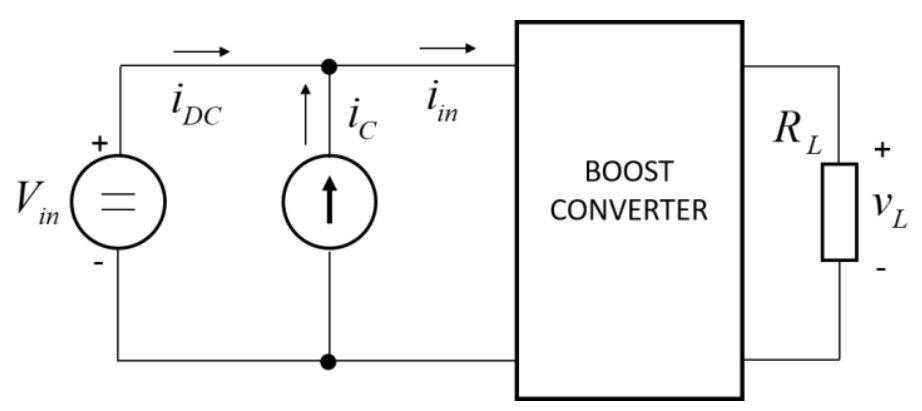

Figure 9. Ideal DM input current compensation for a boost converter

An idealised version of how DM input noise current can be compensated for is given in Figure 9. In this set-up the total input current to the converter $i_{i n}$ is now not only supplied by the DC power source but also a current source $i_{C}$ where:

$$
i_{\text {in }}=i_{D C}+i_{C}
$$

Referring back to Figure $7, i_{\text {in }}$ consists of an average or DC component (that carries the energy for conversion) and a ripple component (that is necessary for the converter to operate). From (5), $i_{D C}$ is the average or DC component only and carries no ripple. The ripple component $i_{C}$ is supplied by the idealised shunt current source.

In practise, $i_{C}$ can be realised by either employing an active source [17] or a shunt capacitor. An active source is usually good for compensating the lower order harmonics in the ripple current but comes at the expense of complexity and cost. More common is the use of a so called DM X-capacitor [17].

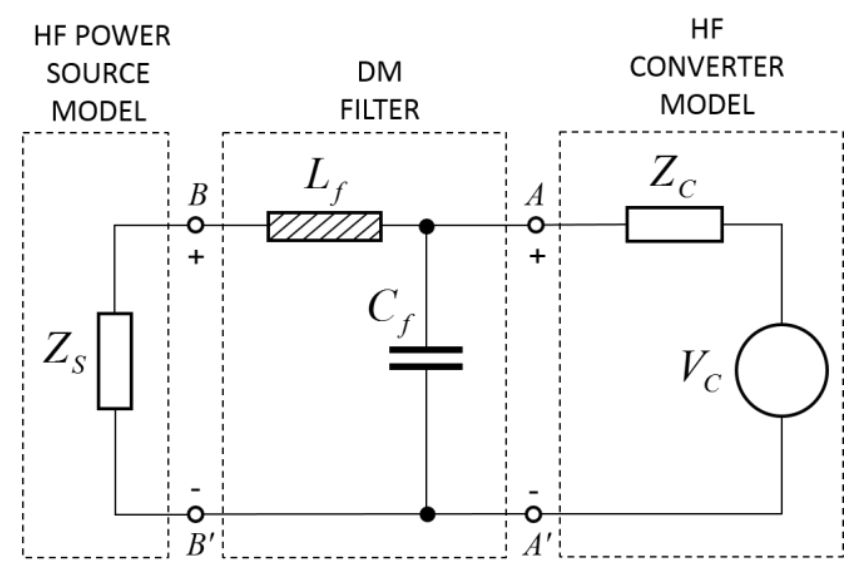

Figure 10. High Frequency (HF) DM noise model for a Boost Converter with LC-filter.

Figure 10 shows a High Frequency (HF) model for a Boost Converter with LC-filter. Note that HF here is in the context of higher harmonics (higher than DC) and therefore includes the converter switching frequency $f_{s}$ and higher frequencies (see Figure 8). In Figure 10, a parallel $C_{f}$ is used to supply (or sink) the ripple current produced by the converter. Whether $C_{f}$ is sinking or supplying ripple (or HF) current is a question of semantics and is determined by the convention of direction chosen for the noise current produced at $V c$. In Figure $9 i_{C}$ is supplied to the converter and in Figure $10, C_{f}$ is sinking noise current. An inductor $L_{f}$ represents the leakage flux in a CM filter choke which is usually deployed with an X-capacitor (see section 4). It can also include some inductance present in the source. Note that Figure 10 is a HF model. If DC only was represented, energy would have flowed from the left of Figure 10 to the right, while EMI generation is represented by $V_{C}$; flowing from the right hand side, crossing $A A^{\prime}$, shunted by $C_{f}$ and blocked by $L_{f}$ to the left hand side noise 
("source") load impedance $Z_{s}$. In a regulatory measurement set-up, $Z_{s}$ would be the HF input LISN impedance (Figure 5).

In Figure 10, the HF noise characteristic of the boost converter is simplified using an equivalent Thevenin source $V_{C}$ and $Z_{C}$.

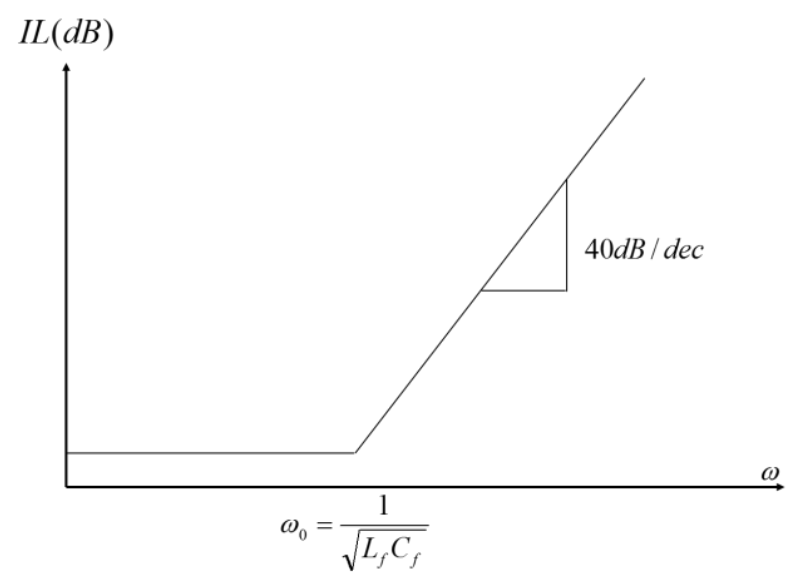

Figure 11. Simplified Insertion Loss (IL) for an LC-filter.

A measure of how well the LC-filter of Figure 10 reduces the boost converter EMI is determined by the filter's Insertion Loss (IL). This is shown in the simplified curve of Figure 11 and is a measure of the EMI loss (in $\mathrm{dB}$ ), with and without the filter.

The break frequency for the LC-filter is:

$$
\omega_{0}=1 / \sqrt{L_{f} C_{f}}
$$

$L_{f}$ is usually fixed or limited to a range so that $C_{f}$ is chosen to make $\omega_{0}$ as low as practically possible. Preferably below the switching frequency. Once again it can be argued that if $L_{f}$ or $C_{f}$ is infinitely large then $\omega_{0}$ will approach zero and the maximum IL will be attained.

In the DM filter of Figure 10, $C_{f}$ cannot be made too large. With a too large capacitance the equivalent series resistance (ESR) and inductance (ESL) in the capacitor becomes a limiting factor in the high frequency response of the capacitor. This will in turn curb the supply of high frequency current that the capacitor can deliver and limit the effectiveness of the filter.

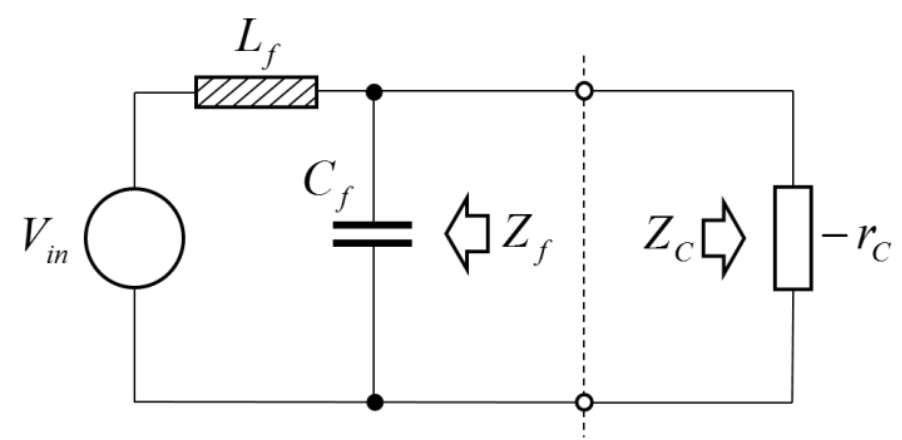

Figure 12. Negative converter impedance vs. source impedance through the LC -filter. .

Another option in making $\omega_{0}$ as low as possible is to make $L_{f}$ as large as possible. This strategy however also has its limits as the converter can become unstable if the source impedance feeding a converter is made too large. This situation is illustrated in Figure 12. The converter has a negative impedance meaning that for constant power output the converter will draw more current as its input voltage is lowered. Referring to Figure 12 , there is a voltage drop at every frequency across $Z_{f}(\omega)$. This voltage drop will become larger as the current being drawn by the converter increases. If this drop is 
too large, the input voltage to the converter will drop and it will become unstable. As a general rule, for converter stability:

$$
\left|Z_{f}(\omega)\right|<<\left|Z_{C}(\omega)\right|
$$

This is known as the Middlebrook stability criterion [18].

In this sub-section it was shown how an LC-filter can reduce the DM EMI of a Boost Converter. There are however, limits to the size of the LC-filter as a too large filter (especially a large $L_{f}$ ) can cause converter instability and a too large $C_{f}$ reduce the ability to respond to high frequency noise.

\section{Common Mode}

In his section it is explained how CM current is generated in a Boost Converter and how it can be mitigated.

Generation of CM current flow is conceptually more difficult to grasp than the generation of DM current as it is parasitic and does not flow as per the conventional functional circuit diagram [19]. The Imbalance Difference Model (IDM) is used to predict CM current noise flow. This is a complete subject on its own [20] and only the results of this modelling method are given here.

Section 4.1. is concluded by three factors that influences the generation of CM noise current in a Boost Converter. These factors can be seen from a CM equivalent IDM (Figure 16). This can be extended to power electronic converters in general.

Section 4.2 contains two general methods of mitigation. Circuit modification and filtering.

\subsection{Common Mode generation in a Boost Converter}

\subsubsection{Common Mode Coupling}

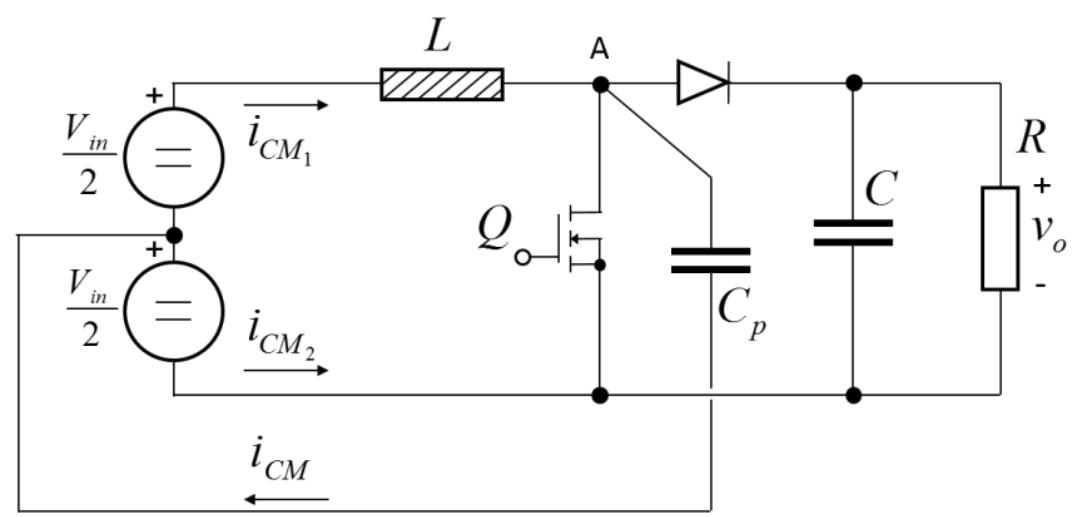

Figure 13. Common Mode coupling and generation in a Boost Converter. From [20].

Figure 13 is used to explain CM current generation and flow in Boost Converter. CM current generation start at point $\mathrm{A}$ in Figure 13. Although there are different parasitic pathways to a common ground (in this case a heat sink), it has been shown that the parasitic capacitance $C_{p}$ has a major influence on CM generation in a Boost Converter [21]. Figure 13 shows an N-channel MOSFET as the switch $Q$. Point A has the largest exposure (capacitive coupling) area to a common ground (outside the functional circuit) as the tab of the MOSFET (drain of $Q$ ) is connected to a heat sink. Furthermore, point $\mathrm{A}$ is a switching node with a high $d v / d t$ swing. If $C_{p}$ is known, the CM current $i_{C M}$ can be obtained from:

$$
i_{C M}(t)=C_{p} \frac{d v_{A}(t)}{d t}
$$


Where $v_{A}(t)$ is similar to the switching waveform of $Q$ as shown in Figure 7. In this case the CM current path return was chosen to be the middle of the source or a reference voltage half of $V_{i n}$. This is somewhat arbitrarily as it is a parasitic path and connect to the circuit at different points. However this $\mathrm{CM}$ reference point is typically chosen as a balanced source simplifies testing and analysis. For example in Figure 5 the common ground or chassis will keep the test set-up balanced if the CM ground is connected to the power source at $V_{\text {in }} / 2$.

The CM current components $\left(i_{C M 1}\right.$ and $\left.i_{C M 2}\right)$ flow in the same direction on the supply power lines. The $\mathrm{CM}$ current $\left(i_{C M}\right)$ return via a common ground, driven by a parasitic capacitance $C_{p}$. Where:

$$
i_{C M}=i_{C M_{1}}+i_{C M_{2}}
$$

Note that it is customary in texts describing general EMI to assume that $i_{C M 1}=i_{C M 2}=i_{C M} / 2$ [23]. This is however not correct as will be shown by implementing the Imbalance Difference Model (IDM) in the next section.

\subsubsection{Imbalance Difference Model}

The Imbalance Difference Model is a way of describing CM generation in circuits with DM EMI. It was first described by Watanabe et al [24] to predict CM noise in digital circuitry. It has been found useful in the understanding of CM generation in power electronics [20].

In its most essential form the IDM is an equivalent CM circuit derived from a DM circuit. This is described in [25]. The IDM describes a "cell" of DM circuitry from which a CM voltage is derived. An equivalent CM circuit (model) is then developed using DM voltage sources as drivers for CM noise current flowing through equivalent impedances, all with respect to a common ground (like a chassis for example).

An example of how a Boost Converter can be converted to its $\mathrm{CM}$ equivalent noise generating circuit is shown next.

Figure 14 shows a complete Boost Converter connected to a LISN with capacitive coupling to a common ground. The converter in Figure 14 is an extension of the one in Figure 13 and includes components in pairs $\left(C_{1}, C_{2}\right.$ and $\left.L_{1}, L_{2}\right)$. From this a DM noise model can be constructed where $Q$ is the primary DM noise driver.

This is shown in Figure 15 where $I_{C}$ is the CM noise current and $h(0 \leq h \leq 1)$ the ratio of CM current distribution flowing between two impedances [25]. For example $h_{L}$ is the ratio or current split of the CM component between $L_{1}$ and $L_{2}$ or:

$$
h_{L}=\frac{L_{1}}{L_{1}+L_{2}}
$$

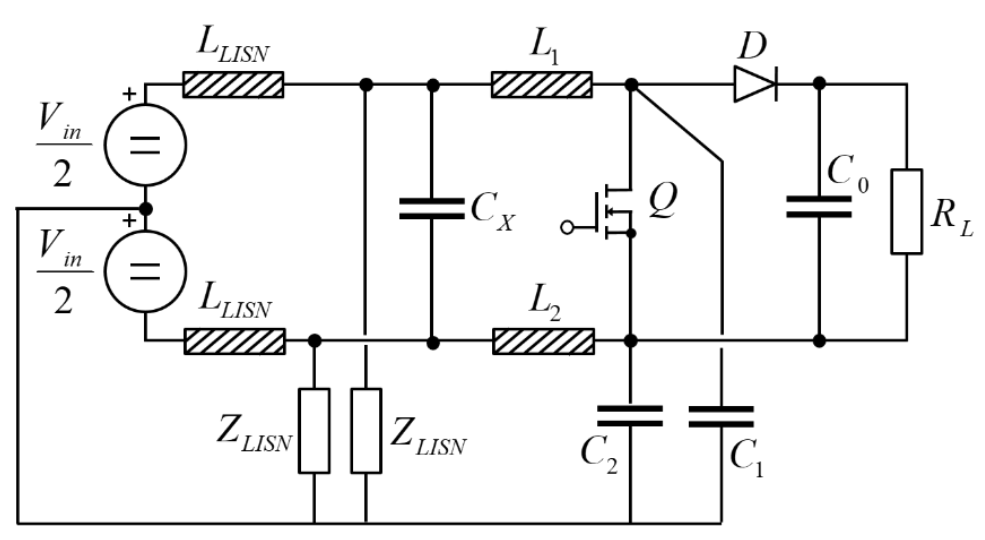

Figure 14. Boost Converter connected to a LISN and showing CM capacitive coupling. From [20]. 


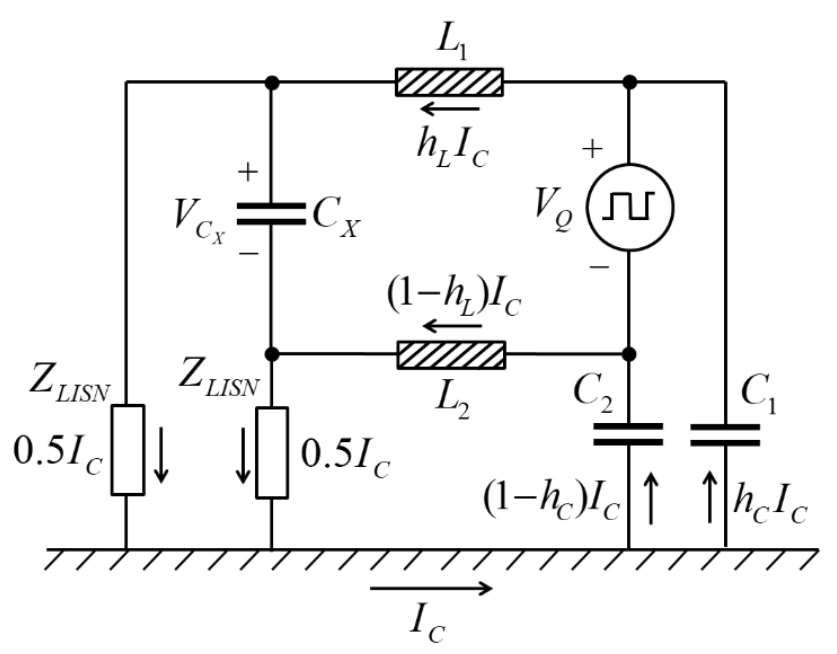

Figure 15. Boost Converter Equivalent Noise Model showing CM current $\left(I_{C}\right)$ flow through the DM circuit. From [20].

According to the IDM [24]:

$$
\Delta V_{C}=\Delta h V_{N}
$$

where $\Delta V_{C}$ is a CM voltage driven by the imbalance difference $(\Delta h)$ around a DM noise source $\left(V_{N}\right)$. The equivalent $\mathrm{CM}$ noise circuit for the DM model in Figure 15 is given in Figure 16.

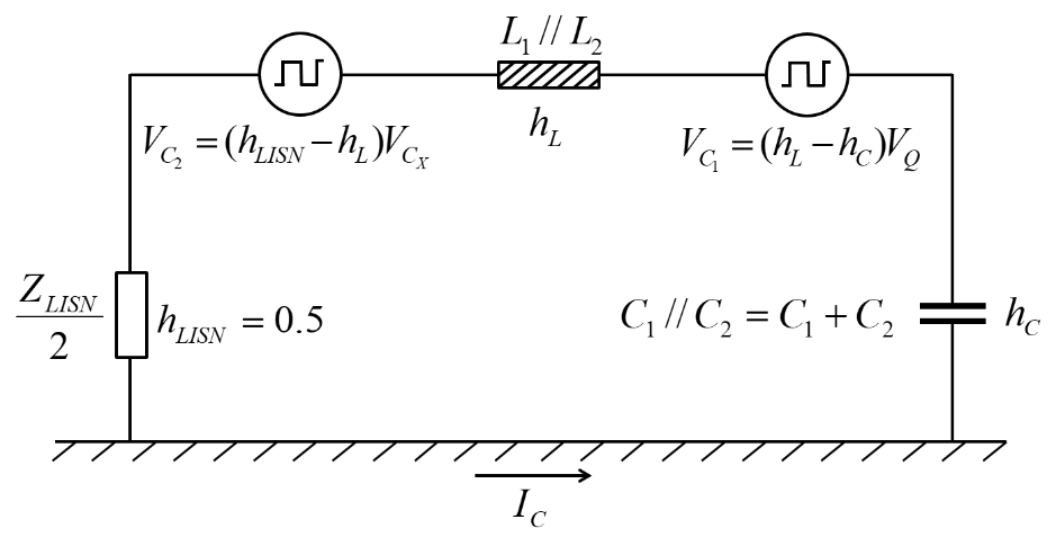

Figure 16. Boost Converter Equivalent CM Imbalance Difference Model (IDM). From [20].

With reference to Figure 16 and [25] it is shown that CM current generation from a Boost Converter is dependent on 3 factors:

- The magnitude of the main power conversion voltage (i.e. the DM signal $V_{Q}$ ) as well as the DM voltage across $C_{x}$.

- The imbalance difference ratios of component values in the DM functional circuit of the Boost Converter (such as the boost inductor $\left(h_{L}\right)$ )

- The impedance of the parasitic couplings between changing voltages in the converter and a common ground (such as a chassis) (for example $C_{1}+C_{2}$ ).

\subsection{Mitigation of Common Mode EMI in a Boost Converter}

In this sub-section, mitigation of CM EMI in a Boost Converter is discussed. First is circuit modifications based on the three factors in section 4.1.2. Second is external CM filtering.

\subsubsection{Circuit Modification}


The CM IDM shown in Figure 16 can be used to explain circuit modifications that will reduce CM noise current.

First is that the magnitude of the DM voltage sources drive the CM noise current. For example $V_{C l}$ where:

$$
V_{C_{1}}=\left(h_{L}-h_{C}\right) V_{Q}
$$

$V_{Q}$ is fixed, but if $\left(h_{L}-h_{C}\right)$ is reduced or zero then no CM voltage will be produced by the switch $Q$ and CM noise current will not flow. In (11), $h_{L}$ and $h_{C}$ are the ratios of current distribution between $L_{1}$ and $L_{2}$ and $C_{1}$ and $C_{2}$ and therefore the ratios of the individual inductance and capacitance values. If these values can be optimized, $\mathrm{CM}$ noise current will be reduced. This however might not be very practical or easy to achieve as $C_{l}$ and $C_{2}$ are parasitic and may vary in individual units of a similar product.

If $h_{L}$ is optimized for (11) then for $V_{C 2}$ :

$$
V_{C_{2}}=\left(h_{L I S N}-h_{L}\right) V_{C_{x}}
$$

and no optimization for $V_{C 2}$ is possible as $h_{L}$ and $h_{L I S N}$ are fixed. One can however optimize between (11) and (12) simultaneously for the smallest values of $V_{C l}$ and $V_{C 2}$.

$V_{C_{x}}$ in (12) is the DM noise voltage across the filter X-capacitor. The larger $C_{x}$ is, the smaller $V_{C_{x}}$ will be. This is consistent with the argument in section 3 where DM filtering was discussed. There is however practical limits in making $C_{x}$ as large as possible. This argument is consistent with the notion that parallel capacitance across the power rails of a converter reduces DM EMI and therefore CM noise current in general.

\subsubsection{Filtering}

In the previous sub-section it was shown how CM EMI can be mitigated by "balancing" of components using the IDM. It was also argued that this cannot completely mitigate all CM noise current. In order to comply with EMI regulations and standards a CM filter has to be employed. This is called an inline power or power line filter. Although deceptively simple from the number of components used, complete texts have been dedicated to this subject [15] as it is not straight forward to design.

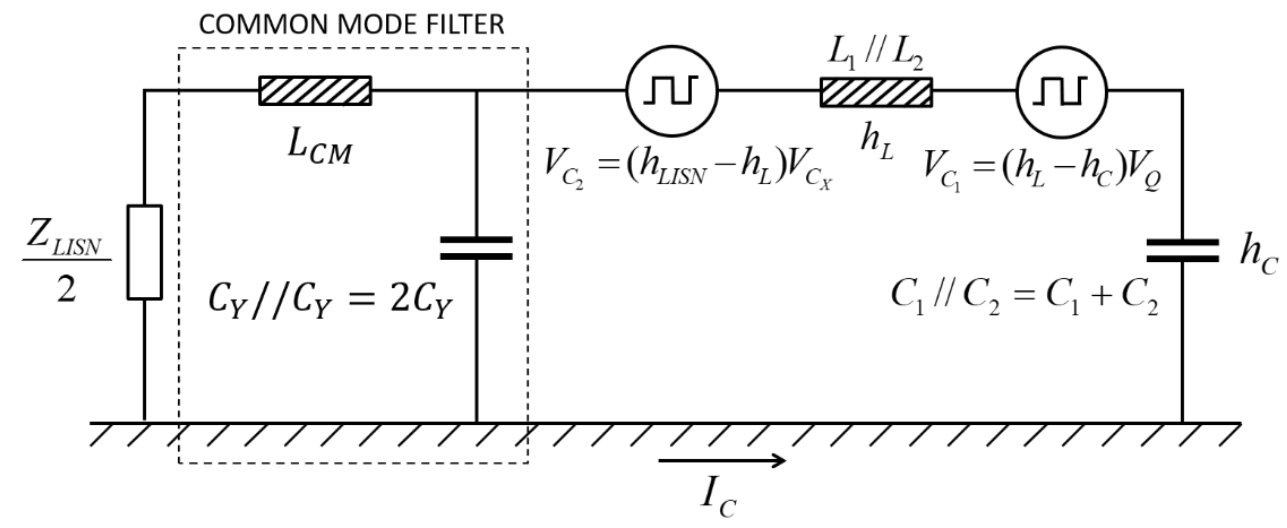

Figure 17. CM filtering of a Boost Converter from an IDM perspective. From [20].

Figure 16 shows an equivalent $\mathrm{CM}$ circuit for a Boost Converter. The $\mathrm{CM}$ noise current is filtered as shown in Figure 17.

The purpose of the CM filter is to keep noise from reaching the LISN impedance $\left(Z_{L I S N}\right)$ as the LISN represent the rest of the supply network that should not be "polluted". To this effect a 
capacitance $\left(C_{Y}\right)$ to a common ground is used to short circuit the CM EMI. This capacitance is in parallel and returns the CM noise current to its source without reaching the LISN (power supply).

Another component, the common mode choke $\left(L_{C M}\right)$ is placed in series and "chokes" the high frequency noise current. Once again keeping the $\mathrm{CM}$ noise current from reaching the supply network.

As with the DM filter in (6), the cut-off frequency $\left(\omega_{0}\right)$ of the CM filter is determined as:

$$
\omega_{0}=1 / \sqrt{L_{C M} \cdot 2 C_{Y}}
$$

The cut-off frequency is chosen to be as low as possible - usually below the switching frequency $f_{s}$. From a theoretical view, $L_{C M}$ and $C_{Y}$ should be as large as possible. This however is not practical as apart from the physical size constraint, parasitic elements limit the high frequency effectiveness of $L_{C M}$ and $C_{Y}$. At very high frequencies and physically large $L_{C M}$ and $C_{Y}$, inter-turn capacitance causes $L_{C M}$ to become capacitive and Equivalent Series Inductance (ESL) causes $C_{Y}$ to become inductive. Thereby "leaking" EMI to the power source.

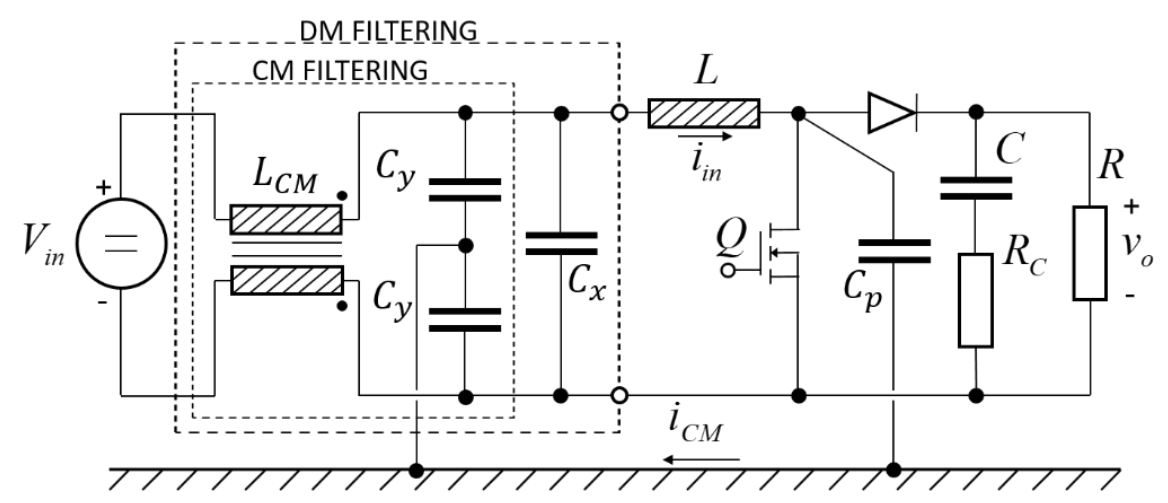

Figure 18. Power line (inline) filter for a Boost Converter.

Figure 17 is an equivalent circuit in $\mathrm{CM}$. The inline filter is realized in DM and shown in Figure 18. In Figure 18, $C_{x}$ is the DM filter capacitance. $C_{Y}$ is the value of one of two capacitors down to a common ground (chassis). These are shown as $\mathrm{CM}$ shunts in Figure 17. Since there are two of $C_{Y}$ in parallel, Figure 17 shows $2 C_{Y}$.

$L_{C M}$ is a special inductor known as a CM choke. A CM choke works by letting through DM current and "choking" or being an inductor in the path of CM.

An example of a CM choke is given in Figure 19. DM flux $\varphi_{D M}$ is created by each winding and cancels out - therefore the inductance to DM current $\left(i_{D M}\right)$ is zero in theory. The CM flux $\varphi_{C M}$ created by the two windings however, adds together to form an inductance in the path of the CM current $i_{C M}$.

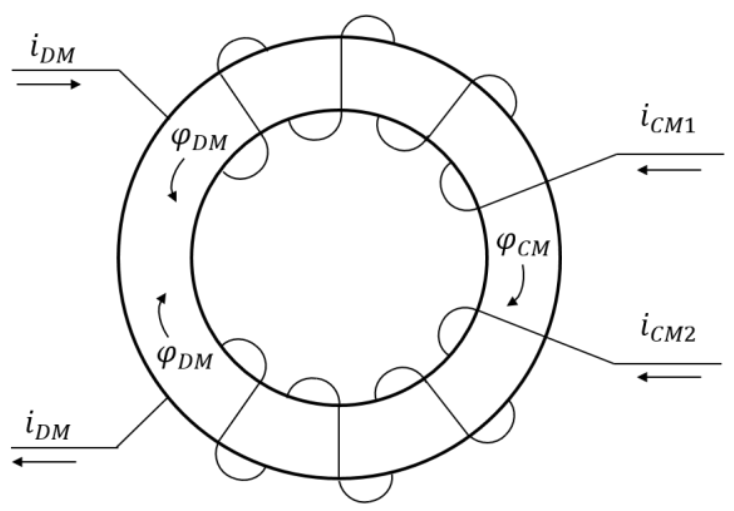

Figure 19. Example of a Common Mode choke. 
In Figure 19, DM inductance is theoretically zero but leakage flux causes a CM choke to exhibit some DM inductance. Therefore in Figure 18, the DM filter consist of the capacitance $C_{X}$ and the leakage inductance of $L_{C M}$. Depending on the coupling, 2 times $C_{Y}$ in series also form part of the DM capacitance. The CM filter is formed by 2 times $C_{Y}$ in parallel and $L_{C M}$.

\section{Experimental Results}

In this section, practical measurements for EMI emanating from a boost converter is given. Two types of mitigation are illustrated. In the first sub-section a power line filter is applied and in the second the converter is balanced according to the IDM.

\subsection{Power Line Filter}

A practical example of the functionality of a power line filter (as seen in Figure 18) is presented in this section. The converter was the same as used to produce the time domain waveforms of Figure 7. Results of measurements showing the noise with and without a filter (as measured from a LISN) are given in Figure 20. The boost converter was balanced (i.e. the boost inductor was split equally into $L_{1}$ and $L_{2}$ as in Figure 14). Capacitive coupling to the chassis was balanced and parasitic only.

Figure 20 shows the background noise with no filter present and no part of the converter energized. Since the measurement was not done in an anechoic chamber, some background noise was present.

As reference the IEC CISPR 14-1 conducted emission limits (for quasi peak measurements) are shown. CISPR 14-1 is required to limit emissions from household appliances, electric tools and similar apparatus.

The converter measurements were peak values and therefore "worst case" and would probably be lower if a quasi peak detector was used. Without the filter the interference exceeds the CISPR 141 limit. With the filter present, the interference is well below the limit.

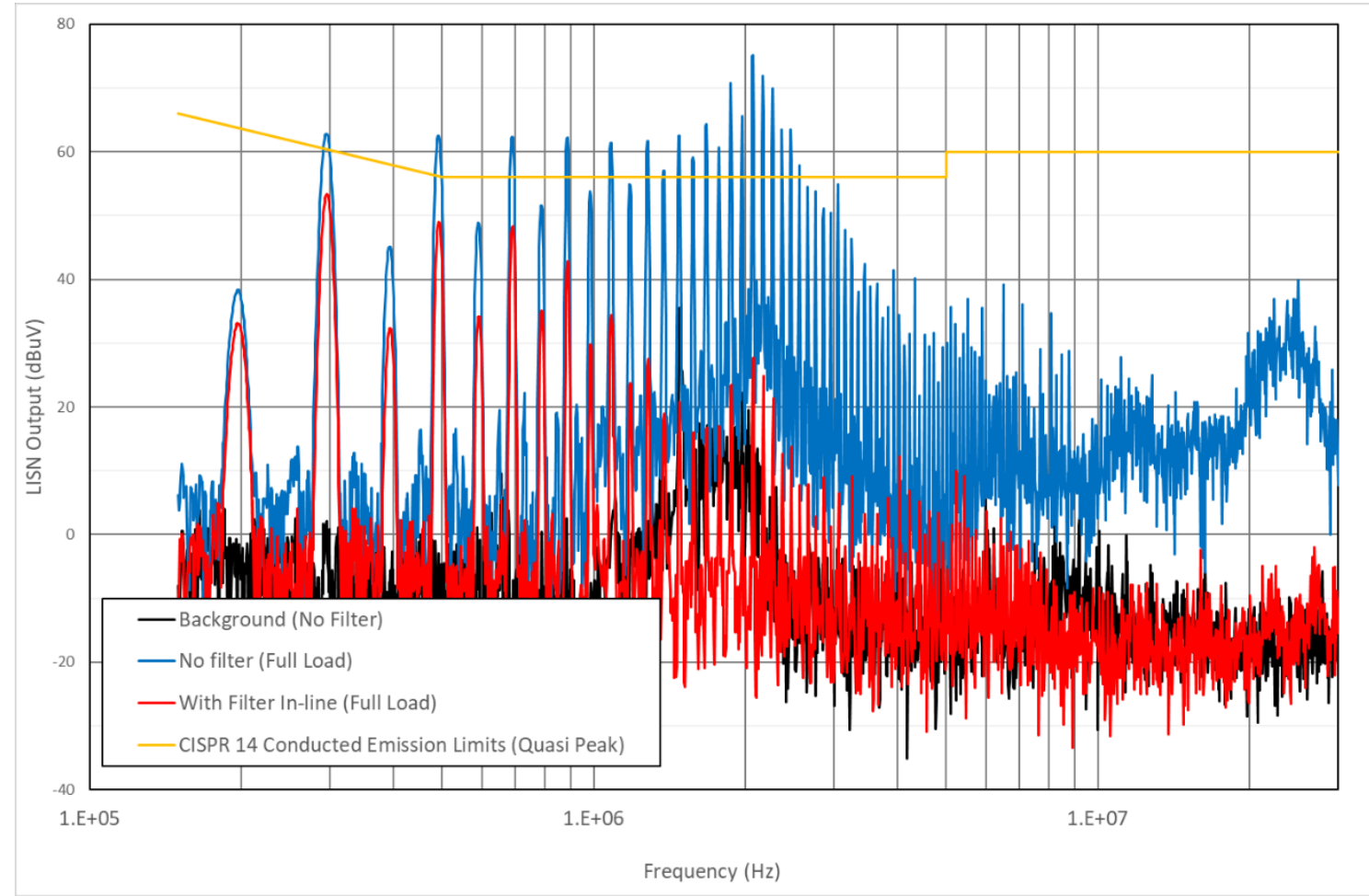

Figure 20. Example of the filtering of power line noise from a balanced boost converter. 


\subsection{Balancing the Converter}

Results for the balancing of a converter is taken from [25]. The converter is shown in Figure 14 and the results in Figure 21. To illustrate the effect of balancing using the IDM, only the CM current is shown in Figure 21. It was measured directly with a current probe on both positive and negative lines simultaneously over the range $10 \mathrm{kHz}-80 \mathrm{MHz}$. This direct measurement of the CM current gives results in $\mathrm{dB} \mu \mathrm{A}$.

Equations (11) and (12) (Figure 16) give expressions for the voltages driving the CM current as seen in Figure 21. Crucial to the two CM voltages being driven by the DM voltages across the switch $\left(V_{Q}\right)$ and X-cap $\left(V_{C x}\right)$ are the factors $\left(h_{L}-h_{C}\right)$ and $\left(h_{L I S N}-h_{L}\right)$. These two factors have a major impact on the CM current of Figure 21. To recap:

$$
h_{\text {LISN }}=0.5 \quad h_{C}=C_{1} /_{\left(C_{1}+C_{2}\right)}=118 /(118+82)=0.59 \quad h_{L}=L_{1} /\left(L_{1}+L_{2}\right)
$$

Table 1. Table showing the factors $\left(h_{L}-h_{C}\right)$ and $\left(h_{\text {LISN }}-h_{L}\right)$ for the traces in Figure 21

\begin{tabular}{ccc}
\hline $\begin{array}{c}\text { Figure } 21 \\
\text { Trace }\end{array}$ & $\left(h_{L}-h_{C}\right)$ & $\left(h_{L I S N}-h_{L}\right)$ \\
\hline (1) & $0 /(0+100)-0.59=-0.59$ & $0.50-0 /(0+100)=0.50$ \\
(2) & $18 /(18+82)-0.59=-0.41$ & $0.50-18 /(18+82)=0.32$ \\
(3) & $50 /(50+50)-0.59=-0.09$ & $0.50-50 /(50+50)=0.00$ \\
(4) & $82 /(82+18)-0.59=0.23$ & $0.50-82 /(82+18)=-0.32$ \\
(5) & $100 /(100+0)-0.59=0.41$ & $0.50-100 /(100+0)=-0.50$ \\
\hline
\end{tabular}

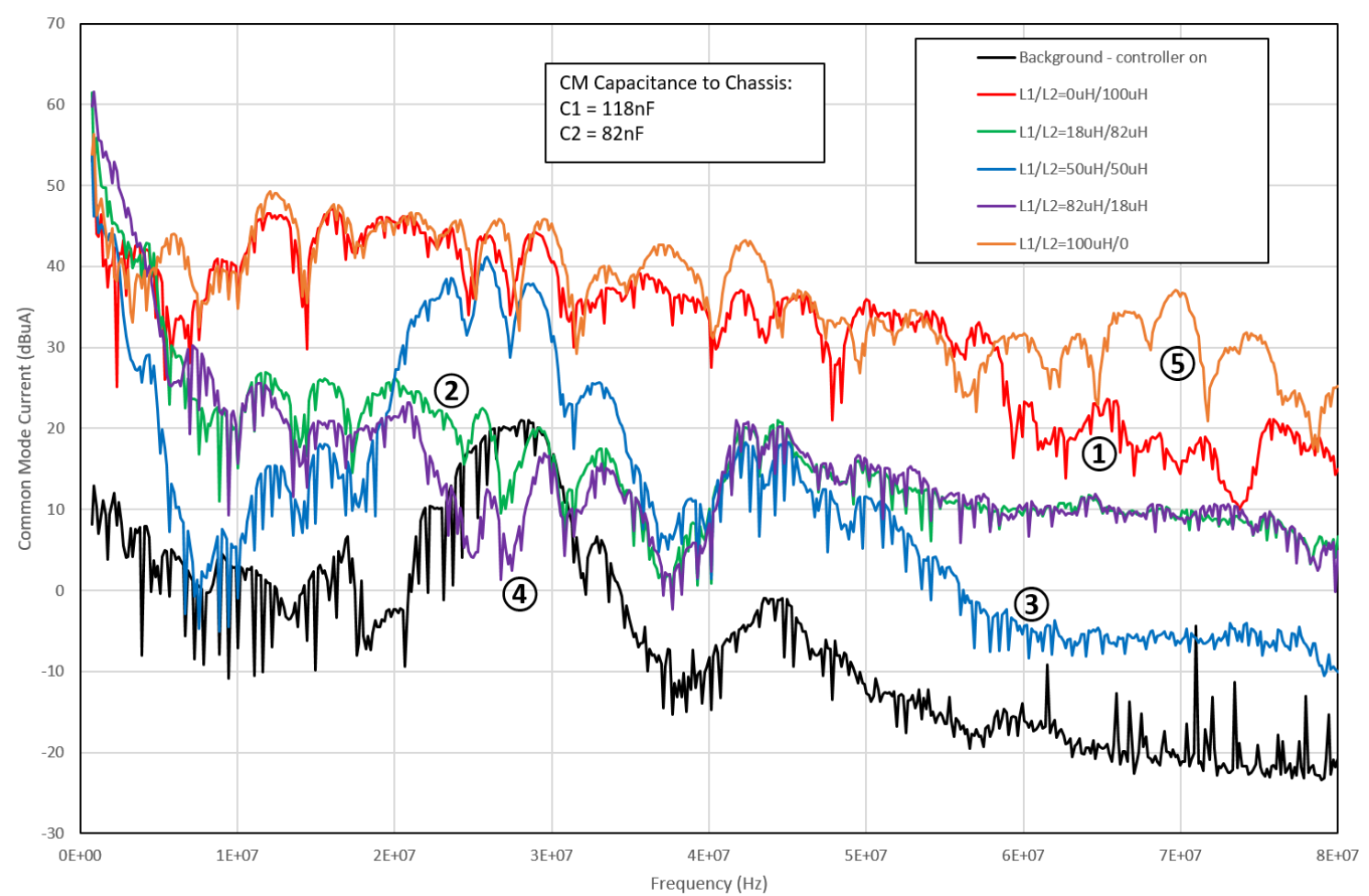

Figure 21. Example of CM current from a boost converter for different boost inductor ratios (L1/L2) given a fixed capacitance ratio to chassis of C1 and C2. See Figure 14. Taken from [25].

Table 1 gives values for the factors $\left(h_{L}-h_{C}\right)$ and $\left(h_{L I S N}-h_{L}\right)$. The larger the absolute values of these factors the higher the CM current. If it is assumed that the DM voltages across the switch $\left(V_{Q}\right)$ 
and X-cap $\left(V_{C x}\right)$ are more or less constant for each variation in total inductance, the measurements correlate with the IDM.

\section{Conclusion}

This paper shows how conducted EMI is generated and mitigated in power electronics converters by using a Boost Converter as an example.

Noise is shown to emanate from converters regardless of the direction of main energy transfer.

Of most concern is noise conducted on the supply power line which carries the EMI to the power source and which can "pollute" other systems.

$\mathrm{CM}$ and DM are introduced as they are the two modes in which EMI is transferred. It is argued that $\mathrm{CM}$ noise in its totality is EMI but that the DM current wave shape drawn by a converter contains both a functional power carrying component and a noise component. The DM noise current component is seen as EMI but is still necessary for the converter to operate.

Measurement for the compliance of a converter to regulations, involve total EMI which is the time domain sum of both CM and DM noise components. Although the total EMI is of concern it can only be mitigated in either CM or DM. This is because the mechanisms for generation and conduction paths for the two modes are different. This difference in the generation, conduction and mitigation is shown in this paper.

The example of a Boost Converter is firstly used to explain DM EMI generation and mitigation by filtering. Design criteria for a DM filter are given.

Secondly the Boost Converter is used to show how CM noise current is generated. This is shown by using the Imbalance Difference Model (IDM). Although the IDM is complicated to the extent of warranting a separate paper, the results of this method are summarized. CM filtering (as part of an inline power filter) are discussed.

The contribution of this study is not only the establishment of a big picture for power converter EMI generation and mitigation but the description of both DM and CM noise origins.

Practical measurements for an in-line power filter is given showing the effect of the filter on the total EMI of a boost converter. Measurements for the CM current produced due to the imbalance difference for different values of the boost conductor are also shown. This shows the validity of the IDM and the mechanism of CM generation.

In conclusion, EMI generation (usually on the supply power lines) of a power electronics converter can be summarized as follows:

- A DM current is supplied to or supplied by a converter. This current contains the main energy transfer wave shape plus an extra "noise" component.

- This extra "noise" component is a function of converter operation without which the converter cannot operate.

- This "noise" component is the DM noise current or DM EMI.

- This DM noise component is the first of three factors that drives the generation of CM EMI.

- The DM wave shape determines the frequency content of the CM noise current.

- A second factor for CM EMI generation is the difference in imbalance in impedances of the CM current flow path through the converter.

- The last factor for CM EMI generation is the magnitude of parasitic coupling to a common ground (chassis).

This paper will be useful to designers seeking the "bigger picture" of how EMI is generated in power converters. It states what can be done to mitigate the noise from both a filtering point of view but also from a generation point of view.

\section{References}

1. van Wyk, J. D.; Lee, F. C. On a Future for Power Electronics. IEEE Journal of Emerging and Selected Topics in Power Electronics 2013, 1 (2), 59-72. https://doi.org/10.1109/JESTPE.2013.2271499.

2. Refaat, S. S.; Ellabban, O.; Bayhan, S.; Abu-Rub, H.; Blaabjerg, F.; Begovic, M. Smart Grid Enabling Technologies; John Wiley \& Sons, 2021. 
3. Bose, B. K.; Wang, F. (Fred). ENERGY, ENVIRONMENT, POWER ELECTRONICS, RENEWABLE ENERGY SYSTEMS, AND SMART GRID. In Power Electronics in Renewable Energy Systems and Smart Grid; John Wiley \& Sons, Ltd, 2019; pp 1-83. https://doi.org/https://doi.org/10.1002/9781119515661.ch1.

4. Zikria, Y. bin; Ali, R.; Afzal, M. K.; Kim, S. W. Next-Generation Internet of Things (IoT): Opportunities, Challenges, and Solutions. Sensors 2021, 21 (4). https://doi.org/10.3390/s21041174.

5. Raj, A.; Steingart, D. Review-Power Sources for the Internet of Things. Journal of The Electrochemical Society 2018, 165 (8), B3130-B3136. https://doi.org/10.1149/2.0181808jes.

6. Santos, D.; Ferreira, J. C. IoT Power Monitoring System for Smart Environments. Sustainability 2019, 11 (19). https://doi.org/10.3390/su11195355.

7. Mishu, M. K.; Rokonuzzaman, Md.; Pasupuleti, J.; Shakeri, M.; Rahman, K. S.; Hamid, F. A.; Tiong, S. K.; Amin, N. Prospective Efficient Ambient Energy Harvesting Sources for IoT-Equipped Sensor Applications. In Electronics; 2020; Vol. 9. https://doi.org/10.3390/electronics9091345.

8. O W, N. M.; Undeland, T. M.; Robbins, W. P.; Wiley, J. POWER ELECTRONICS Converters, Applications, and Design THIRD EDITION; 2003.

9. Rodrigues, E. M. G.; Godina, R.; Pouresmaeil, E. Industrial Applications of Power Electronics. Electronics 2020, 9 (9). https://doi.org/10.3390/electronics9091534.

10. de Beer, A. S. Determination of Power Components in Nonlinear Electrical Networks, 1990.

11. de Beer, A. S.; Wooding, G. N.; van Wyk, J. D. Problematic Aspects When Using a LISN for Converter EMI Characterisation. In Proceedings of the IEEE International Conference on Industrial Technology; 2013; pp 633637. https://doi.org/10.1109/ICIT.2013.6505745.

12. Costa, F.; Magnon, D. Graphical Analysis of the Spectra of EMI Sources in Power Electronics. IEEE Transactions on Power Electronics 2005, 20 (6), 1491-1498. https://doi.org/10.1109/TPEL.2005.857564.

13. Bryant, B.; Kazimierczuk, M. K. Voltage-Loop Power-Stage Transfer Functions with MOSFET Delay for Boost PWM Converter Operating in CCM. IEEE Transactions on Industrial Electronics 2007, 54 (1), 347-353. https://doi.org/10.1109/TIE.2006.885136.

14. Mitchell, D. M. Power Line Filter Design Considerations for Dc-Dc Converters. IEEE Industry Applications Magazine 1999, 5 (6), 16-26. https://doi.org/10.1109/2943.798333.

15. Nave, M. J. Power Line Filter Design for Switched-Mode Power Supplies; 2010.

16. Domínguez-Palacios, C.; González-Vizuete, P.; Martín-Prats, M. A.; Mendez, J. B. Smart Shielding Techniques for Common Mode Chokes in EMI Filters. IEEE Transactions on Electromagnetic Compatibility 2019, 61 (4), 1329-1336. https://doi.org/10.1109/TEMC.2019.2918863.

17. Mainali, K.; Oruganti, R. Conducted EMI Mitigation Techniques for Switch-Mode Power Converters: A Survey. IEEE Transactions on Power Electronics 2010, 25 (9), 2344-2356. https://doi.org/10.1109/TPEL.2010.2047734.

18. Middlebrook, R. D. Input Filter Considerations in Design and Application of Switching Regulators. In IAS'76; 1976.

19. de Beer, A. S. Problematic Concepts in the Introduction of EMC. In 2008 IEEE International Symposium on Electromagnetic Compatibility; 2008; pp 1-4. https://doi.org/10.1109/ISEMC.2008.4652120.

20. de Beer, A. Reducing Common Mode EMI Generation in a Boost Converter Using the Imbalance Difference Model. In 2017 IEEE International Conference on Environment and Electrical Engineering and 2017 IEEE Industrial and Commercial Power Systems Europe (EEEIC / I CPS Europe); 2017; pp 1-6. https://doi.org/10.1109/EEEIC.2017.7977484.

21. Yao, J.; Lai, Y.; Ma, Z.; Wang, S. Advances in Modeling and Reduction of Conducted and Radiated EMI in Non-Isolated Power Converters. In 2021 IEEE Applied Power Electronics Conference and Exposition (APEC); 2021; pp 2305-2312. https://doi.org/10.1109/APEC42165.2021.9487164.

22. Paul, C. R. Introduction to Electromagnetic Compatibility; John Wiley \& Sons, 2006; Vol. 184.

23. Watanabe, T.; Wada, O.; Miyashita, T.; Koga, R. Common-Mode-Current Generation Caused by Difference of Unbalance of Transmission Lines on a Printed Circuit Board with Narrow Ground Pattern. IEICE transactions on communications 2000, 83 (3), 593-599.

24. de Beer, A. S.; Faul, K. Factors Influencing Common Mode EMI Generation in Boost Converters. In 2018 IEEE 4th Global Electromagnetic Compatibility Conference (GEMCCON); 2018; pp 1-5. https://doi.org/10.1109/GEMCCON.2018.8628475. 